
internationales

vol. 26 - $n^{\circ} 1 \mid 2010$

Les médias des minorités ethniques

\title{
Gildas SIMON, La planète migratoire dans la mondialisation
}

Guy Di Méo

\section{(2) OpenEdition}

1 Journals

Édition électronique

URL : https://journals.openedition.org/remi/5051

DOI : 10.4000/remi.5051

ISSN : $1777-5418$

Éditeur

Université de Poitiers

\section{Édition imprimée}

Date de publication : 1 février 2010

Pagination : 187-191

ISBN : 978-2-911627-54-5

ISSN : 0765-0752

\section{Référence électronique}

Guy Di Méo, "Gildas SIMON, La planète migratoire dans la mondialisation », Revue européenne des migrations internationales [En ligne], vol. 26- $n^{\circ} 1$ | 2010, mis en ligne le 30 août 2010, consulté le 14 avril 2022. URL : http://journals.openedition.org/remi/5051 ; DOI : https://doi.org/10.4000/remi.5051 


\section{NOTES DE LECTURE}

SIMON Gildas

La planète migratoire dans la mondialisation.- Paris, Armand Colin, collection U, 2008.- 255 pages.

ISBN : 978-2200346799

Gilivi ildas Simon consacre donc un nouveau livre à la question qui le tient en haleine depuis plus d'une trentaine d'années, à savoir celle des migrations internationales. Ces pages empreintes de sagesse et de compréhension profonde, d'empathie pour tous les migrants du monde, très bien écrites, passionnantes d'un bout à l'autre, témoignent d'une immense culture et revêtent une incontestable originalité. Gildas Simon inaugure ici une approche novatrice des migrations abordées à petite échelle. Il se place pour cela dans le paradigme de la mondialisation et produit une réflexion à la fois synthétique et exigeante. Ainsi, la référence permanente que fait l'auteur à la "planète migratoire » affirme-telle, dans son texte, "la prévalence des logiques planétaires sur les modes de fonctionnement locaux ou régionaux».

On ne s'étonnera guère que ce pionnier des analyses migratoires en géographie, fondateur du laboratoire MIGRINTER de Poitiers, renouvelle de la sorte des études et des réflexions largement nourries par les travaux de terrain de cette (son) équipe, si dynamique et si inventive. Dans un contexte postmoderne qui réfute les valeurs universelles, Gildas Simon pose, à juste titre et un peu à contre-courant, le principe de l'existence de réels universaux. Il pense que ceux-ci justifient plus d'un mouvement migratoire. Il parie qu'ils motivent plus d'un migrant quittant sa terre. À ce propos, Gildas Simon rappelle toutefois, d'une part, que "l'immense majorité des populations du monde, y compris dans les pays du Sud, ne souhaite pas partir, ne souhaite pas devoir émigrer ». Mais il redit aussi, d'autre part, combien les migrations s'efforcent de répondre à quelques-uns des besoins les plus fondamentaux de la personne humaine ; essentiellement lorsqu' elle ne peut pas, ou ne peut plus les satisfaire dans une aire territoriale de proximité. Ces besoins c'est, par exemple : échapper à la faim et disposer d'une alimentation correcte (quantitativement et qualitativement), se loger dans de bonnes conditions, pouvoir se soigner et faire face aux imprévus de la vie, être traité avec équité dans son contexte social et territorial... Bref, bénéficier d'un accès légitime $a u$ "développement humain». Ainsi, par-delà les inévitables différenciations culturelles des sociétés du monde, des représentations universelles et constantes du bien-être existent bel et bien, partagées par toutes et tous, quelle que soit leur origine, comme le rappelle à bon escient Gildas Simon.

Outre ce constat réconfortant, l'un des grands mérites de l'ouvrage est d'assumer une rupture épistémologique majeure. G. Simon y parvient au prix du dépassement d' une stricte segmentation analytique des faits de migration observés, méthode traditionnelle qu'il rejette pour privilégier une prise en considération globale des déplacements à caractère migratoire qu'il étudie. Dans cette optique, l'auteur abandonne la notion un peu restrictive "d'espace migratoire " qui servit, jadis, d'outil méthodologique à ses premières recherches (entre Tunisie et France en particulier). Il lui préfère, plus opératoire aujourd' hui, la notion de " champ migratoire "; soit "l'ensemble de l'espace transnational unissant (...) lieux d'origine, de transit et d'installation », autrement dit "l' espace parcouru et structuré par des flux stables et réguliers de migrations et par l'ensemble des flux (matériels et idéels) induits par la circulation des hommes ». Malgré les explications avancées par Gildas Simon, il faut bien avouer qu'un tel outil se distingue assez mal de modèles voisins, voire concurrents, comme le " territoire circulatoire » proposé par Alain Tarrius. Mais qu'importe! D'une part, il faut bien admettre que le mot "circulatoire " manque de précision. D'autre part, le terme de " champ migratoire » décrit bien ces combinaisons géographiques et culturelles très fluides, cristallisées un instant par les migrations (ce ne sont pas des circulations banales) 
qui les parcourent. Ces combinaisons sont sujettes à d'innombrables recompositions spatiales, quelle que soit leur fréquente inscription dans la longue durée des temps historiques ; à l'image, par exemple, des diasporas.

Mais il y a plus; mieux encore que celui de territoire, circulatoire ou pas, le terme " champ », emprunté à l'outillage conceptuel de Pierre Bourdieu, évoque bien la présence en ces espaces d'éléments structurants variés, mais non déterminants pour des acteurs (les migrants en l'occurrence) somme toute compétents, maîtres au final de leur décision de migrer ou pas... À l'exception bien sûr de ces migrants forcés, objets et victimes de tous les trafics, sorte d'esclaves modernes présents et présentes jusque dans nos villes. Ajoutons que cette idée de "champ » traduit, de façon tout à fait heureuse, une mise sous tension d'espaces investis, dans le tissu même de leurs structures matérielles, par des "charges symboliques, porteuses pour le migrant d'espoirs, d'utopies et de mythes, d'imaginaires »... Soit autant d'éléments renvoyant au vécu le plus intime des sujets migrants ; vécus sans lesquels on ne saurait comprendre la force de cet élan qui pousse chaque année, sur nombre de chemins anciens et nouveaux du monde, plusieurs dizaines de millions (près de deux centaines en fait) d'individus. Parmi eux, on compte de plus en plus de femmes, d'étudiants, d'entrepreneurs transnationaux et de travailleurs aux qualifications très différenciées, de mineurs isolés, de demandeurs d'asiles... Au total des personnes fragiles, vulnérables, exploitées... Mais pas seulement. Des pauvres, sans doute, mais aussi et de plus en plus des migrants instruits, formés, à la recherche de réussite et d'idéal, poussés par un espoir d'ascension sociale et de "réalisation de soi ", comme l'écrit Gildas Simon.

"Les migrations ont réalisé la première mondialisation de la terre des hommes », celle qui a accompagné (entre - 200000 et - 5000 avant notre ère) leur dissémination sur les différents continents. Les migrations, de plus ou moins grande ampleur, ne sont donc pas nouvelles, et Gildas Simon en administre la preuve. Ce qui est plus neuf, à le lire, c'est la relation intime qui unit désormais cette mobilité des humains (du Sud vers le Nord, mais aussi dans le cadre respectif des Nords et des Suds) aux mutations profondes qui affectent les économies nationales (désormais transnationales) et les territoires (de moins en moins clos, mais toujours plus urbanisés, voire métropolisés) de la planète. Ce qui change aussi, c'est l'imbrication intime (coconstitutive?) de ce mouvement avec la circulation accélérée et sans frontière des flux d'informations, d'idées, de biens, de capitaux...

La planète migratoire s'inscrit parfaitement dans le libéralisme triomphant et dans sa mondialisation protéiforme, quel que soit le décalage des idéologies, des mentalités et des systèmes politiques. Si les migrations d'échelle planétaire redistribuent des fonds énormes et indispensables dans les contrées appauvries du Sud, elles stimulent et conditionnent la richesse du Nord. Pour Gildas Simon, les pouvoirs politiques des pays riches (et derrière eux leurs populations) qui maltraitent trop souvent les immigrants, font preuve d'un véritable autisme. Les crises conjoncturelles et le court terme masquent en effet (mais les opinions et les pouvoirs en sont-ils vraiment dupes?) ce qui constitue, en fait, un formidable phénomène de régulation économique et de dynamisme culturel à l'échelle de la planète. À ce dernier titre, les hybridations nées des rencontres interculturelles ne favorisent-elles pas la création artistique et scientifique, pour peu que l'on parvienne à élaborer un vivre ensemble digne de ce nom? De plus, alors que s'impose partout (idéologiquement sinon effectivement) la logique libérale des marchés, l'ordre politique revient à la vieille stratégie des empires d'antan. C'est celle des marches et des glacis chargés de refouler de soi-disant envahisseurs, pourtant essentiels au fonctionnement du système global (cas de l'espace de Schengen?). N'est-ce pas eux, en effet, ces étrangers (l'autre) trop souvent honnis, qui vont assurer l'apport indispensable de populations jeunes et actives, dans ces pays où les nationaux vieillissent? N'est-ce pas eux qui sont et seront susceptibles de combler les déficits enregistrés par l'offre de travail sur 
des marchés de l'emploi souvent déséquilibrés? Sans parler de ces nécessaires solidarités internationales, convoquées dans la perspective de la promotion d'une planète durable!

Ce n'est pas si fréquent qu'un ouvrage de géographie parvienne à associer la rigueur concrète des terrains et des exemples avec une réflexion transdisciplinaire d' une grande profondeur. Celle-ci porte à la fois sur les causes profondes des migrations d'aujourd'hui et sur le sens de ces migrations en rapport avec une nouvelle conception de l'espace devenu mobile par son interférence avec les champs migratoires qui le parcourent et le structurent en forme de kaléidoscope. Dans le premier registre, Gildas Simon ne néglige pas plus les raisons psychologiques et sociales les plus secrètes, celles qui ressortissent aussi de l'imaginaire et du mythe, que celles qui tiennent aux contraintes très lourdes de la survie alimentaire etlou physique. Dans le second, il saisit de quelle façon la structuration des champs migratoires fournit sa trame à la nouvelle toile planétaire ; ceci dans un monde territorialisé qui se déconstruit et se recompose selon des figures éphémères et volatiles.

Mais en définitive, mieux que de commenter ce livre, il convient d' urgence de le lire.

Guy Di MÉO

UMR 5185 ADES

Université de Bordeaux - CNRS

PIAN Anaïk

Aux nouvelles frontières de l'Europe. L'aventure incertaine des Sénégalais au Maroc.- Paris, La Dispute, 2009.$237 \mathrm{p}$.

ISBN : 978-2-84303-161-8

Celon un rapport de Médecins sans frontières (2005) - cité par l'auteur entre six et quinze mille migrants subsahariens en situation irrégulière seraient au Maroc dont les principales nationalités seraient représentées par les Nigérians, Maliens suivis des Sénégalais et Congolais. L'ouvrage nous enseigne des expériences migratoires de Sénégalais transitant par le Maroc avec comme enjeu principal le passage clandestin en Europe. C'est précisément dans cette optique que les migrants originaires d'Afrique subsaharienne - très souvent mis en exergue dans la lutte contre l'immigration irrégulière au Maroc - élaborent de nouvelles façons de voyager afin de contourner les dispositifs répressifs mis en place. Mais Anaïk Pian nous fait surtout découvrir les relations sociales complexes qui s'établissent entre les aventuriers, terme utilisé par l'auteur pour désigner ces migrants sénégalais qui circulent et migrent à la marge, si ce n'est dans l'illégalité. Elle montre également les différentes articulations qui s'opèrent entre l'externalisation des contrôles migratoires et les mobilités des aventuriers bloqués au Maroc. Et elle termine avec une partie de ceux dont la destination finale envisagée s'est éloignée.

Cette forme de migration vers l'Europe qu'est l'aventure se déploie en effet sur le mode d'une migration par étapes, en ce sens dit-elle, qu'elle se confronte à des défis successifs, aux termes desquels chaque palier franchi scelle une épreuve et en annonce une autre. Que ce soit par la Mauritanie, le Mali voire le Niger et la Libye puis l'Algérie ou directement en avion de Dakar à Casablanca, les aventuriers sénégalais usent de multiples réseaux, assemblés entre eux; très loin de l'idée d'organisations centralisées, ces réseaux relient ainsi de nombreux territoires et diverses expériences migratoires. Ces parcours qui défient les logiques territoriales et souveraines des nations, viennent «buter » sur l'externalisation du contrôle européen des frontières qui s'opère au Maroc; processus qui est en train de perturber tout un système d'échanges inter africains, souligne l'auteur. Pour preuve, l'union européenne ne cesse de demander au Maroc d' imposer l'obligation de visa à l'ensemble des ressortissants des pays subsahariens, espérant "endiguer " une partie de ces mobilités.

Face au durcissement de ces politiques européennes auquel le Maroc est de plus en plus associé, sont apparus des "foyers »qui sont des éléments de stabilité dans l'instabilité 
de l'aventure. Ces hébergements communautaires dirigés par un Thiaman ${ }^{l}$ peuvent accueillir également des Maliens, Gambiens, Guinéens... et se caractérisent par leur dénuement; Anaïk Pian rappelle ainsi que ce sont tout d'abord des lieux de passage où les populations se recomposent sans cesse. En conséquence, au sein de ces "communautés d'itinérance »(Claire Escoffier), les rapports entre les migrants se caractérisent à la fois par des solidarités pragmatiques et des solidarités marchandisées, avec des sentiments d'appartenance à un même groupe et des distances critiques à l'égard de celui-ci. $E t$ l'auteur détaille tout un ensemble de règles existant au sein de ces "foyers" qui sont d'autant plus importantes qu'elles invitent à remettre en cause la criminalisation de la figure du «passeur ». Enfin dans ces lieux d'attente forcée, aux inquiétudes perpétuelles d'être raflés par la police, s'ajoute la peur d'être reconduit aux frontières du Maroc, notamment vers l'Algérie à proximité d'Oujda. C'est ainsi que l'aventure côtoie la "migrerrance ", autre néologisme ${ }^{2}$ qui exprimerait à la fois l'espérance de réussir sa "migration" et l'errance subie.

Pas plus que les autres régions du Maroc, les régions entourant les présides de Ceuta et Melilla ne sont pas officiellement interdites aux aventuriers, toutefois elles assignent à celles et ceux qui s'y trouvent - ou qui ne devraient pas s'y trouver, selon la police marocaine - une identité particulière. Juste avant le passage des frontières, les campements informels de Bel Younes ou Mesnara - situés au Nord du Maroc - assurent comme les "foyers", une fonction protectrice en dépit de leur vulnérabilité. À l'appui des travaux de l'anthropologue Roger Martin

1 Souvent les thiamen logent à l'extérieur des "foyers", notamment quand ils ont accumulé suffisamment d'argent, après avoir réussi de nombreux passages vers l'Europe.

2 Gérard Sadik in Migreurop (2009) Atlas des migrants en Europe. Géographie critique des politiques migratoires, Paris, Armand Colin, $144 \mathrm{p}$. Ce néologisme fait écho à celui de « déserrance » conçu par Claude-Valentin Marie et réutilisé par Anaïk Pian.
Keesing, l'auteur souligne que ces campements constituent des systèmes de résistances aux politiques étatiques tout en mimant certains aspects de ce pouvoir dominant. Ainsi des règles sont établies en référence aux institutions contemporaines, avec l'emploi de termes comme "nations unies » ou " casques bleus »...; ces derniers ayant notamment comme rôle d'accompagner les migrants qui partent franchir les barbelés des deux enclaves espagnoles. En parallèle des " conventions » collectives et écrites cadrant leur quotidien, et découlant de l'accumulation d'une expérience migrante commune, il y a aussi toutes les débrouillardises liées à l'institutionnalisation du passage, titre du sixième chapitre. Ainsi face à la délocalisation des contrôles européens les empêchant de circuler légalement, les aventuriers construisent leurs propres règles de voyage. La voie "clandestine » qui conduit vers l'Europe est appréhendée non pas comme un acte illégal ou illégitime, mais comme un acte légitime, se justifiant par la non-obtention de visa.

Pour les autres n'ayant pas réussi, certains ont des troubles psychologiques liés à l'errance; leur dénuement physique et moral leur laisse peu de chance dans cette vie devenue un cauchemar. Ce point n'est pas développé, et on le regrette, à la différence des deux derniers "moyens pour sortir de l'aventure » que sont le retour au pays et la reconversion dans les réseaux commerçants sénégalais. Pour en arriver à l'une de ces deux décisions, il a fallu ce temps de maturation et d'accumulation pour que la personne ait vraiment le sentiment d'une limite $^{3}$. La durée indéterminée du voyage, l'échec du passage, l'argent reçu du pays devenant un poids, le sentiment d'enfermement, sont autant de facteurs qui imposent le choix d'un autre projet de vie. Pour ceux qui retournent au Sénégal, ils "s'insèrent »le temps d'un aller simple dans le commerce à la valise, défini comme un principe de «solidarité

3 Claire Bidard (2006) Crises, décisions et temporalités autour des bifurcations biographiques, Cahiers internationaux de Sociologie, vol. CXX, p. 40 (cité par l'auteur). 
contrainte et confiance obligée ». Par exemple, pour celles et ceux qui restent, l'achat d'une carte de séjour ${ }^{4}$ permet à certains Sénégalais de s'insérer dans le commerce. Mais leur situation demeure précaire, elle s'apparente à un combat quotidien pour (sur)vivre; et à cela s'ajoutent les discriminations : jets de cailloux, refus de s'asseoir à coté d'eux dans les bus, continuer à avancer pour ne pas être blessé alors que des paroles désobligeantes vous sont proférées.

Jusqu'où peut-on parler de transit lorsque le passage - vers l'Europe en l'occurrence - se fait de plus en plus difficile? La mobilisation de notions telles l'entre-deux - expression empruntée à Violette Rey - de zone tampon, de nasse ou d" "espace extraterritorial ${ }^{5}$ où

4 « Le recours à la corruption devient un capital de mobilité indispensable » écrit l'auteur ; tactiques qui se retrouvent à toutes les échelles, de l'achat d'un billet de train aux passages des frontières.

5 En référence au no man's land de 14 kilomètres situé aux frontières de l'Algérie. l'arbitraire est souvent de mise, souligne à quel point des aventuriers se retrouvent prisonniers après qu' au fil des mois voire des années, leur chance de parvenir en Europe se trouve compromise.

Enfin on soulignera les entretiens présents tout au long de l'ouvrage qui illustrent judicieusement les vies des aventuriers sénégalais vivant aujourd' hui au Maroc. Ces interviews restituées parfois sous formes de simples conversations ou dialogues, sont de très belles images écrites de la sociologie compréhensive; car au-delà de décrire les parcours migratoires de ces aventuriers, elles permettent aussi de comprendre les enjeux des contrôles migratoires qui s'opèrent sur le territoire marocain entre l'Union européenne, le Maroc et d'autres États africains. Anaïk Pian offre ainsi une contribution de qualité à travers de nombreux portraits, et nous apporte une indispensable réflexion sur l'expérience d'une mobilité bloquée en cours de route.

Olivier CLOCHARD

ADES (Bordeaux 3), associé à Migrinter

Errata : À la page 32 de l'ouvrage, une ultime reformulation de l'éditeur aboutit à un contresens, il faut lire "le fait que les aventurières sénégalaises rencontrées furent peu nombreuses est à l'image de leur présence relativement réduite sur les routes de l'aventure ». Enfin Ghadamès est une ville frontalière située à l'ouest de la Libye (p. 71) et les camps représentés sur les deux cartes (pp. 76-77) sont ceux de Bel Younes et Mesnara. 